\title{
Evidence for a subcortical contribution to intracortical facilitation
}

Running title: A subcortical origin of intracortical facilitation

Authors: *Patrick Wiegel ${ }^{1,2},{ }^{*}$ Niclas Niemann ${ }^{1,2}, \ddagger$ John C. Rothwell ${ }^{4} \& \ddagger$ Christian Leukel ${ }^{1,2,3}$

${ }^{1}$ Department of Sport Science, University of Freiburg, Germany

${ }^{2}$ Bernstein Center Freiburg, University of Freiburg, Germany

${ }^{3}$ Freiburg Institute for Advanced Studies (FRIAS), University of Freiburg, Germany

${ }^{4}$ Sobell Department of Motor Neuroscience and Movement Disorders, University College London, WC1N 3BG London, United Kingdom

*equal contribution

‡equal contribution

Number of pages: 20; Figures: 3

Total number of words in (i) the whole manuscript: 3,787; (ii) the abstract: 182

Corresponding author:

Christian Leukel

Department of Sport Science

University of Freiburg

Schwarzwaldstr. 175

79117 Freiburg - Germany

Email: christian.leukel@sport.uni-freiburg.de

Phone: + 49-761-203-4521

Fax: $+\underline{49-761-203-4534}$

Keywords: transcranial magnetic stimulation; H-reflex; corticospinal; paired-pulse; motor cortex 


\section{Abstract}

Intracortical facilitation (ICF) describes the facilitation of an EMG response (motor evoked potential) to a suprathreshold pulse (S2) of transcranial magnetic stimulation (TMS) by a preceding subthreshold pulse (S1) given 10 to $15 \mathrm{~ms}$ earlier. ICF is widely assumed to originate from intracortical mechanisms. In the present study, we used spinal H-reflexes to test whether subcortical mechanisms can also contribute to the facilitation. Measurements were performed in the upper limb muscle flexor carpi radialis (FCR) in 17 healthy volunteers, and in the lower limb muscle soleus (SOL) in 16 healthy volunteers.

S2 given alone facilitated the H-reflex. When S1 preceded S2 by $10 \mathrm{~ms}$, the amount of facilitation increased, compatible with ICF. However, S1 given alone also facilitated the H-reflex, suggesting that it had evoked descending activity even though its intensity was well below resting motor threshold. Across participants, the amount of $\mathrm{H}$-reflex facilitation from $\mathrm{S} 1$ alone was proportional to the degree of H-reflex facilitation with combined S1-S2.

These results indicate that subcortical mechanisms can contribute to ICF and potentially add to the variability of the ICF measure reported in previous studies. 


\section{Introduction}

Intracortical facilitation (ICF) refers to the interaction between two transcranial magnetic stimulation (TMS) pulses, S1 and S2, applied consecutively to the primary motor cortex. S2 at suprathreshold intensity evokes a compound muscle potential (motor evoked potential, MEP) in the target muscle. S1 given alone is subthreshold and evokes no MEP. If both S1 and S2 are applied with an interstimulus interval (ISI) of 10 to $15 \mathrm{~ms}$ then the S2 MEP is facilitated (Kujirai et al., 1993). Although ICF is widely believed to originate from the interaction of neurons within motor cortex activated by S1 and S2 (Ziemann et al., 1996; Ziemann et al., 2015), spinal mechanisms have additionally been considered to play a role (Di Lazzaro et al., 2017). One argument supporting this idea relates to the observation that despite clear facilitation of MEPs there was no sign of an increased amplitude of the accompanying descending activity recorded from electrodes in the epidural space of the human spinal cord (Di Lazzaro et al., 2006). Here we asked whether subcortical mechanisms contribute to ICF. Several previous studies have demonstrated that subcortical activity can be evoked with TMS intensities below the conventionally defined (Rossini et al., 2015) resting motor threshold (RMT) (Day et al., 1989; van der Linden \& Bruggeman, 1993; Niemann et al., 2017) and even active motor threshold (Hanajima et al., 2007). Consequently, corticospinal activity from S1-S2 may be influenced by a "subthreshold" $\mathrm{S} 1$ evoking a small descending volley that increases subcortical excitability and increases the susceptibility to S2. In order to test this, we applied H-reflexes to probe for changes in the recruitment of spinal motoneurones during ICF.

\section{Materials and Methods}

\section{Experiments and subjects}

Two separate measurements were performed for the upper limb muscle flexor carpi radialis (FCR) $(\mathrm{N}=17$, three females) and for the lower limb muscle soleus (SOL) $(\mathrm{N}=16$, five females). These measurements were an extension of a larger study that was recently published in the Journal of Neurophysiology (Niemann et al., 2017), focussing on the noninvasive assessment of D- and I-waves from TMS rather than on mechanisms of ICF. All tested 
subjects were healthy, had no contraindications to TMS (Rossini et al., 2015), and were aged between 23 and 27 years. Nine of the subjects participated in both, the FCR and SOL measurements. The FCR and SOL measurements in those subjects were conducted on different days with a minimum of 48 hours in between measurements. The order of measurements was randomized across subjects. All participants gave written informed consent to the procedures. The study was approved by the local ethics committee of the AlbertLudwigs-University in Freiburg (423/15).

\section{Electromyography (EMG)}

Surface EMG (EISA, Pfitec Biomedical Systems, Endingen, Germany) was recorded from the left FCR or the left SOL using bipolar surface electrodes (Blue sensor P, Ambu®, Bad Nauheim, Germany) placed $2 \mathrm{~cm}$ apart over the muscle belly. The preference for the left side was due to the arrangement of the setup. A ground electrode was placed at the caput ulnae (in experiments on FCR) and at the tibial plateau (in experiments on SOL). Impedance was below $10 \mathrm{k} \Omega$. EMG signals were pre-amplified (x 100), further amplified (2x), bandpass filtered $(10-1300 \mathrm{~Hz})$ and sampled at $2 \mathrm{kHz}$.

\section{Electrophysiological stimulation techniques}

The subjects were seated in a custom-built laboratory seat with headrest. The legs were placed on a footboard in an outstretched (around $160^{\circ}$ to $170^{\circ} \mathrm{knee}$ angle) and relaxed position. The left arm was flexed and pronated and placed on the subjects' lap. Subjects wore a forearm bandage which was stabilized with tape mounted to the chair (only in experiments on FCR).

\section{Peripheral nerve stimulation (PNS)}

H-reflexes were elicited with a constant current stimulator (DS7a, Digitimer ${ }^{\circledR}$, Hertfordshire, UK) by stimulating the median nerve approximately $1-3 \mathrm{~cm}$ proximal to the elbow joint (experiments in FCR) and the posterior tibial nerve at the popliteal fossa (experiments in SOL). 
Stimuli consisted of square wave-pulses of $0.2 \mathrm{~ms}$ duration (median nerve) and $0.5 \mathrm{~ms}$ duration (tibial nerve). Further details can be found in (Niemann et al., 2017).

Transcranial magnetic stimulation (TMS)

Single-pulse and paired-pulse TMS were applied over the contralateral primary motor cortex hand/arm area (experiments on FCR) and leg area (experiments on SOL) using a Magstim® $200^{2}$ stimulator with a BiStim unit (Magstim ${ }^{\circledR}$ Company Ltd., Whitland, UK) and a 70-mm figure-of-eight batwing coil for experiments in SOL, and a more focal 50-mm figure-of-eight coil for experiments in FCR. The handle of the coil was mounted to a stand that was positioned on top of the chair (Manfrotto $\AA$ Magic Arm, Lino Manfrotto \& Co, Cassola, Italy). Brainsight TMS navigation (Brainsight $2 \AA$, Rogue Research, Montreal, Canada) was used to monitor the position of the coil relative to the skull.

The optimum site for stimulation was defined as the site where clear MEPs could be evoked with the lowest possible stimulation intensity. For FCR, the coil was held tangentially on the scalp at an angle approximately $45^{\circ}$ to the mid-sagittal plane with the handle pointing laterally and posteriorly (inducing a posterior-anterior directed current). For SOL, the coil was placed tangentially on the scalp, the handle pointed posteriorly at an angle of $0^{\circ}$ with respect to the midline (inducing a posterior-anterior directed current).

RMT was determined as the minimum stimulator output (in \% of maximum stimulator output, MSO) required to evoke MEPs of $\sim 50 \mu \mathrm{V}$ in at least three out of five consecutive trials applied at the same intensity (Rossini et al., 2015).

\section{Conditioned H-reflexes by TMS}

Conditioning of H-reflexes with TMS was applied in accordance with previous studies (e.g. Nielsen et al., 1993; Leukel et al., 2012). The objective is to promote coincidence of TMS-evoked activity and afferent activity by PNS at the spinal level. The ISI at which this occurs varies from muscle to muscle depending on the conduction times in the afferent 
connections and the corticospinal connections. Negative ISIs indicate that PNS precedes TMS and positive ISIs indicate the opposite.

ISls of $-6 \mathrm{~ms}$ to $+8 \mathrm{~ms}$ (experiments in FCR) and $-5 \mathrm{~ms}$ to $+8 \mathrm{~ms}$ (experiments in SOL), in 1 ms steps, were tested in the present study. For all measurements, electrical stimulation was adjusted at an intensity to evoke $\mathrm{H}$-reflexes of 15 to $25 \%$ of the respective maximum M-wave (Mmax) (Crone et al., 1990), on the upsloping part of the H/M recruitment curve. TMS was applied with an intensity of $115 \%$ of RMT, adjusted to the nearest $1 \%$ of MSO $(116.7 \pm 1.0 \%$ of RMT in FCR and $113.1 \pm 1.0 \%$ of RMT in SOL).

Intracortical facilitation (ICF)

ICF-effects on spinal H-reflexes were tested by adding a subthreshold TMS pulse (S1) that preceded the suprathreshold TMS pulse (S2) by 10 ms (Kujirai et al., 1993).

The intensity of the conditioning S1 was the same as used in our recent publication (Niemann et al., 2017), namely $69.4 \pm 1.3 \%$ of RMT in FCR and $67.5 \pm 1.0 \%$ of RMT in SOL, which is similar to that applied in previous studies testing ICF (Biabani et al., 2018).

\section{Conditioned $\mathrm{H}$-reflex protocols}

Fifteen H-reflexes were recorded at each ISI for each of the three conditions: S2 (baseline condition), S1, and S1-S2. Fifteen MEPs (S2, S1, S1-S2) and fifteen unconditioned H-reflexes were also recorded. All conditions were intermixed and pseudo-randomized in one experimental protocol as described in (Niemann et al., 2017). The delay between subsequent stimuli was always $4 \mathrm{~s}$ to avoid changes in post-activation depression of the $\mathrm{H}$-reflex (Crone \& Nielsen, 1989).

\section{Data analyses and statistics}

Peak-to-peak amplitudes of all electrophysiological responses were calculated from the unrectified FCR and SOL EMG. Root-mean-square (RMS) values of the first millisecond (1 ms) from the unrectified $\mathrm{H}$-reflex onset were calculated in all trials and averaged, for conditions S2 
and S1-S2, at early facilitation delays (EFDs, see next paragraph) $-1 \mathrm{~ms}$ and $0 \mathrm{~ms}$, and also for unconditioned H-reflexes, respectively. H-reflex onset was visually determined based on the plot of superimposed H-reflexes from all trials (Figure 1). A clear determination of the onset by this approach was possible in every tested subject. Before calculating RMS values, in each trial we corrected for a potential offset of the baseline EMG by matching the value at $\mathrm{H}$-reflex onset to zero. Data points subsequent to $\mathrm{H}$-reflex onset (at $0.5 \mathrm{~ms}$ and at $1 \mathrm{~ms}$ following $\mathrm{H}$ reflex onset) were corrected according to the offset correction performed at H-reflex onset.

We use the term ISI to refer to the interval between delivery of S1 and S2. In the Results section, these timings have been corrected to take into account individual peripheral and central conduction delays, such that 0 ms indicates the instant at which the fastest conducted peripheral afferent volley reached spinal motoneurones at the same time as the fastest conducted corticospinal volley. Negative intervals (i.e. $<0 \mathrm{~ms}$ ) indicate that the fastest conducted afferent volley arrives first whereas positive intervals (i.e. $>0 \mathrm{~ms}$ ) indicate that the fastest conducted corticospinal volley arrived first. We refer to these adjusted intervals as EFDs to distinguish them from ISIs.

In order to determine the time of coincident arrival of the fastest conducted peripheral and descending volley, we identified the onset of $\mathrm{H}$-reflex facilitation (early facilitation) in each participant (Leukel et al., 2015; Taube et al., 2015; Niemann et al., 2017). We therefore computed uncorrected paired Student's t-tests between peak-to-peak amplitudes of S2-conditioned $\mathrm{H}$-reflexes at each of the negative ISIs and the unconditioned $\mathrm{H}$-reflex. The first interval (from more negative to less negative ISIs) at which there was a significant $(p<.05)$ increase in the size of the conditioned H-reflexes compared to the unconditioned H-reflexes was denoted as EFD =0 ms (Figure 1). In two measurements (both in SOL), the statistical tests yielded no significant result because the amount of facilitation at early time intervals was weak. In these measurements, we denoted the early facilitation based solely on visual inspection of the conditioned H-reflex plot like in earlier studies (Taube et al., 2015). In each 
participant, mean peak-to-peak amplitude conditioned $\mathrm{H}$-reflexes at each EFD were referenced to the mean of the unconditioned $\mathrm{H}$-reflex and expressed as the percentage.

Two-way repeated measures ANOVAs were performed separately for FCR and SOL on the percent $\mathrm{H}$-reflex facilitation (based on the peak-to-peak amplitudes) with factors TMS PULSE (S2, S1-S2) and EFD (FCR: 13; SOL: 10). The factor EFD for FCR contained all intervals from EFD $-2 \mathrm{~ms}$ to EFD $+10 \mathrm{~ms}$. For SOL, the factor EFD encompassed all intervals from EFD 0 ms to EFD +9 ms. These were time intervals with no missing values from any participant. Missing values in SOL measurements resulted in case the early facilitation occurred at a more negative ISI than $-3 \mathrm{~ms}$ or a more positive ISI than $-2 \mathrm{~ms}$. This was the case in four subjects, where the early facilitation occurred at ISI $-1 \mathrm{~ms}$ (two subjects), at ISI $-5 \mathrm{~ms}$ and at ISI $-4 \mathrm{~ms}$, respectively.

Spearman's rank-order correlations were computed for peak-to-peak amplitudes as well as $1 \mathrm{~ms}$ RMS data at time points EFD $-1 \mathrm{~ms}$ and EFD $0 \mathrm{~ms}$, separately for FCR and SOL. The data used for correlations were individual differences of peak-to-peak amplitudes/RMS values (mean values of 15 trials) between the S1-S2 condition and the S2 condition (i.e. peak-to-peak amplitude/RMS value S1-S2 minus peak-to-peak amplitude/RMS value S2, calculated at the corresponding EFD).

Paired Student's t-tests were performed for all other a-priori and post-hoc analyses. Results obtained from multiple comparisons were corrected by the Benjamini-Hochberg procedure (Benjamini \& Hochberg, 1995).

The level of significance was set to $p<.05$ for all tests. Mean values and standard error of the mean (SEM) are reported. Greenhouse-Geisser corrected values for ANOVAs are reported in case sphericity of the tested samples was violated (Mauchly's test). Data were statistically analysed with SPSS software 24.0 (SPSS $\AA$, Chicago, IL, USA). 


\section{Results}

H-reflex facilitation by TMS

$\mathrm{S} 2$ alone facilitated the $\mathrm{H}$-reflex for 11 milliseconds (from EFD $0 \mathrm{~ms}$ to EFD $+10 \mathrm{~ms}$ ) in both muscles, as described previously (Niemann et al., 2017) (Figure 2). Facilitation of H-reflexes was greater by S1-S2 than by S2 alone. The repeated-measures ANOVA showed for FCR a significant main effect for TMS PULSE $\left(F_{1,16}=17.272, p=.001\right)$ and EFD $\left(F_{2.3,36}=13.29\right.$, $p<.001)$, but no significant interaction for TMS PULSE $x \operatorname{EFD}\left(F_{5.2,82.8}=0.972, p=.441\right)$. For SOL, the repeated-measures ANOVA showed a main effect for TMS PULSE $\left(F_{1,15}=6.072, p=.026\right), \operatorname{EFD}\left(F_{2.8,42.6}=3.077, p=.040\right)$ and a significant interaction for TMS PULSE $x \operatorname{EFD}\left(F_{4.6,68.9}=2.71, p=.031\right)$. Post-hoc analyses of the peak-to-peak amplitudes with paired Student's t-tests (results displayed in Figure 2) revealed that H-reflexes in FCR were significantly more facilitated by $\mathrm{S} 1$-S 2 than by $\mathrm{S} 2$ at EFDs $-2,0,+2,+4,+5$ and $+11 \mathrm{~ms}$. $\mathrm{H}$-reflexes in SOL were significantly more facilitated by S1-S2 than by $\mathrm{S} 2$ at EFDs $-1,0$, and $+2 \mathrm{~ms}$.

Although the intensity of S1 was subthreshold, S1 produced significant facilitation of the $\mathrm{H}$-reflex peak-to-peak amplitude in FCR. The mean facilitation across EFDs $0 \mathrm{~ms}$ to $+9 \mathrm{~ms}$ compared to the unconditioned H-reflex amplitude was $111 \pm 5 \%(p=.035)$. In SOL, this comparison did not reveal a significant facilitation $(p=.10)$, despite the fact that the mean value was higher than $100 \%(111 \pm 6 \%$ of the unconditioned $\mathrm{H}$-reflex).

In the S1-S2 condition, H-reflex amplitudes in FCR and SOL were facilitated at EFD -1 ms, at a time before arrival of the earliest volley from S2 (i.e. EFD $=0 \mathrm{~ms}$ ). This could be because the fastest conducting volley from S2 facilitated the late part of the $\mathrm{H}$-reflex, and this may have increased the H-reflex peak-to-peak amplitude covering this late part. The duration of the $\mathrm{H}$-reflex is several milliseconds and it is caused by afferents with different conduction times (Burke, 2016). At EFD -1 ms, the fastest conducting S2 volley can thus coincide with afferents with slower conduction times at the spinal motoneurones. To test whether $\mathrm{S} 1$ actually 
facilitated the H-reflex at EFD -1 ms, we calculated the initial $1 \mathrm{~ms}$ RMS value of the H-reflex from its onset, which should be uncontaminated by any input from S2. When analysed in this way, the FCR H-reflex was facilitated at EFD $=-1 \mathrm{~ms}$ by $123 \pm 8 \%$ compared to the $1 \mathrm{~ms}$ RMS values of unconditioned H-reflexes $(p<.001)$ whereas in SOL it was $129 \pm 15 \%(p<.001)$. This facilitation was indeed caused by $\mathrm{S} 1$, which was triggered $9 \mathrm{~ms}$ earlier. Across participants, the amount of facilitation by S1-S2 compared to S2 at EFD -1 ms correlated with the amount of facilitation at EFD $0 \mathrm{~ms}$ (i.e. at the arrival time of $\mathrm{S} 2$ at the spinal level, corresponding to the onset of ICF) (FCR: $r=0.498, p=.042$ ); SOL: $r=0.76, p=.001$ ) (Figure 2). When using the peak-to-peak amplitudes instead of the 1 RMS values for this analysis, in FCR the correlation between EFD -1 ms and EFD 0 ms was no longer significant $(r=.45, p=$ $.073)$, in contrast to SOL $(r=0.79, p<0.001)$.

MEP and H-reflex

S1-S2 MEPs were significantly higher than S2 MEPs in both muscles (FCR: $p=.0129$; SOL: $p=.0133)$ (Figure 3).

Control H-reflex size was $1.07 \pm 0.20 \mathrm{mV}$ in FCR and $1.05 \pm 0.10 \mathrm{mV}$ in SOL. H-reflex latency was $16.6 \pm 0.4 \mathrm{~ms}$ in FCR and $30.6 \pm 0.4 \mathrm{~ms}$ in SOL.

\section{Discussion}

The results of the present study indicate that S1 in FCR produced subcortical activity which facilitated the H-reflex. The correlation between the amount of FCR H-reflex facilitation from only $\mathrm{S} 1$ and the H-reflex facilitation with $\mathrm{S} 1-\mathrm{S} 2$ suggests that this subcortical activity contributed to ICF.

In FCR, as expected, S2 alone facilitated the H-reflex. The facilitation started at EFD $0 \mathrm{~ms}$, indicating the time point when the fastest conducted corticospinal S2-volley arrived at the spinal level (Nielsen et al., 1993; Di Lazzaro et al., 2008; Di Lazzaro et al., 2012; Niemann et al., 2017). S1 alone also significantly facilitated the H-reflex, confirming earlier reports about corticospinal effects and facilitation of H-reflexes produced with subthreshold TMS (Day et al., 
1989; Nielsen et al., 1993; van der Linden \& Bruggeman, 1993; Guzman-Lopez et al., 2012). The facilitation started when the fastest conducted volley from S1 reached the spinal level (at EFD $0 \mathrm{~ms}$ ) and it lasted over a period of at least $11 \mathrm{~ms}$ (EFD $0 \mathrm{~ms}$ to EFD $+10 \mathrm{~ms}$ ). This $\mathrm{H}-$ reflex facilitation from $\mathrm{S} 1$ is evident in the S1-S2 condition, namely at the time points preceding EFD $0 \mathrm{~ms}$, i.e. EFD $-2 \mathrm{~ms}$ and $-1 \mathrm{~ms}$, respectively. Note that, in the $\mathrm{S} 1-\mathrm{S} 2$ condition, EFD $0 \mathrm{~ms}$ reflects the time point when the fastest conducting volley from S2 reached the spinal level, and that $\mathrm{S} 1$ was applied $10 \mathrm{~ms}$ before S2.

The initial part (1 ms from H-reflex onset) of the $\mathrm{H}$-reflex facilitation from $\mathrm{S} 1$-S2 compared to S2 alone was correlated between EFD -1 ms and EFD $0 \mathrm{~ms}$, suggesting that the subcortical effect of S1 contributed to the S1-S2 facilitation that took place at EFD $0 \mathrm{~ms}$. We propose that the fastest conducting S2 volley (at EFD $0 \mathrm{~ms}$ ) recruited more spinal motoneurones when S1S2 were combined because S1 produced EPSPs of spinal motoneurones and thus made it easier for S2 to trigger action potentials. In principle, S1 did have sufficient time (10 ms at EFD $0 \mathrm{~ms}$ ) to interact with other spinal circuits, including presynaptic inhibition of afferent fibres mediating the H-reflex, la reciprocal inhibition, and Ib inhibition (Pierrot-Deseilligny \& Burke, 2005). However, modulation of these circuits by S1 would likely show a different modulation of the H-reflex than what we observed. The H-reflex should be depressed and not facilitated if increased la reciprocal inhibition via recruitment of la inhibitory interneurons of the antagonistic muscle would have been the main cause (Cowan et al., 1986). A depressed and not facilitated H-reflex would likely be expected if changes in presynaptic inhibition at la terminals by S1 would be an important mechanism (Meunier, 1999). In this earlier study (Meunier, 1999), presynaptic inhibition of la afferent terminals to FCR motoneurones was reported to be increased by TMS. It is furthermore unlikely that reduced Ib inhibition from S1 was responsible for the H-reflex facilitation that we observed, because this facilitation would be expected to be weak and equivocal (lles \& Pisini, 1992; Pierrot-Deseilligny \& Burke, 2005) and not pronounced and consistent (across subjects) like in our experiments. Thus, the most likely explanation of our results is an increase of spinal motoneurone excitability by $\mathrm{S} 1$. We note that this is still a proposition, and future studies may evaluate changes in spinal motoneurone 
excitability with direct measures to test this prediction.

It is interesting that only the correlation of the $1 \mathrm{~ms}$ RMS values between EFD $-1 \mathrm{~ms}$ and EFD 0 ms was significant, but not the correlation of the peak-to-peak amplitudes. This may suggest that the subcortical effect of $S 1$ was causally related to only the fastest conducting connections (corticospinal and/or la afferent). Only approximately the first millisecond of the $\mathrm{H}$-reflex origins from fast conducting monosynaptic la afferent input, slower conducting monosynaptic and also disynaptic connections contribute to the later part (Marchand-Pauvert et al., 2002; Burke, 2016). Further, only the first millisecond of the conditioned H-reflex is influenced by the fastest conducting, presumably monosynaptic corticospinal connections (Nielsen et al., 1993; Nielsen et al., 1995). In contrast to the 1 ms RMS value which covers only the initial part of the H-reflex, the peak-to-peak amplitude covers also the later part of the H-reflex. Thus, connections that contribute to that later part may not (or only weakly) be affected by $\mathrm{S} 1$.

The facilitation of $\mathrm{H}$-reflexes occurred not only at EFD 0 ms but also at more positive EFDs. In particular, H-reflex facilitation was observed at EFDs $+2 \mathrm{~ms},+4 \mathrm{~ms},+5 \mathrm{~ms}$, and $+11 \mathrm{~ms}$. This disjointed collection of time points may indicate that several yet not specified mechanisms were responsible for the facilitation. The delay between S1 and S2 $(10 \mathrm{~ms})$ is sufficiently long for $\mathrm{S} 1$ to recruit several spinal and also cortical circuits. At the spinal level, interactions between S1 and S2 could have occurred at spinal motoneurones, propriospinal neurones, and also segmental interneurons (Pierrot-Deseilligny \& Burke, 2005). At the level of the brain, effects could have occurred between neurons within the primary motor cortex (Ziemann et al., 1996; Chen et al., 1998). Further, effects could origin from interactions between the primary motor cortex and neighbouring connected areas like the premotor cortex (Dum \& Strick, 2005) and between the primary motor cortex and subcortical areas like the reticular formation (Baker, 2011). It will be the task of future studies to elaborate on the possibly multiple mechanisms underlying the facilitatory effects from S1. 
In SOL, we observed a significant facilitation of the H-reflex by S1-S2 compared to S2 like in FCR. However, unlike in FCR, it is not possible to conclude that part of this facilitation was subcortical in nature. Our analysis did not demonstrate evidence of a significant facilitation of the H-reflex by S1. Possibly, spinal mechanisms like reciprocal inhibition from the antagonistic muscle TA prevented the facilitation from being significant, by counteracting the corticospinal activity produced by S1 (Cowan et al., 1986). In addition, our data do not provide convincing evidence that S1 was causally related to the S1-S2 facilitation, as in FCR. The significant correlation of the initial facilitation of the H-reflex by S1-S2 compared to S2 between EFD -1 ms and EFD 0 ms (both RMS values and also peak-to-peak amplitudes) were biased by an outlier (data for peak-to-peak amplitudes were not shown). Taken together, the results in SOL may point towards a subcortical interaction between S1 and S2, but the potentially stronger bias from spinal circuitries than in FCR prevents us from making conclusions.

Another problem with SOL was that in two subjects the early facilitation could not be unequivocally defined like it was possible in FCR. In fact, in these participants we had to define the early facilitation by visual means because the amount of facilitation of the early time intervals was weak and the statistical tests yielded no significant result. This presumably lower impact of synaptic input at the spinal motoneurones from fastest conducting corticospinal volleys in SOL compared to FCR (Lemon et al., 2002) clearly constitutes a methodological problem when determining the early facilitation.

In conclusion, our experiments in FCR provide evidence that subcortical mechanisms contributed to ICF. It is important to note that these are unlikely to be the sole mechanism for ICF. Indeed, there is likely to be a mixture of effects, from subcortical and cortical sources (Cash et al., 2017). The main message, however, is that subcortical interactions can occur in commonly applied ICF paradigms. The importance of a subcortical contribution may vary e.g. between individuals and different diseases. They may also contribute to the variability of the ICF measure seen in many reports. 


\section{Acknowledgements}

This work was supported by the German Research Foundation (LE 2744/7-1).

\section{Competing interests}

There are no conflicts of interest.

\section{Data accessibility}

Raw data can be accessed upon request by contacting the corresponding authors.

\section{Author contributions}

N.N., P.W., J.C.R., and C.L. conceived and designed research; N.N. and P.W. performed experiments; N.N., P.W., and C.L. analysed data; N.N., P.W., J.C.R., and C.L. interpreted results of experiments; N.N., P.W. and C.L. prepared figures; J.C.R. and C.L. drafted manuscript; N.N., P.W., J.C.R., and C.L. edited and revised manuscript; N.N., P.W., J.C.R., and C.L. approved final version of manuscript.

\section{Abbreviations}

ICF: intracortical facilitation

TMS: transcranial magnetic stimulation

MEP: motor evoked potential

ISI: interstimulus interval

RMT: resting motor threshold

FCR: flexor carpi radialis muscle

SOL: soleus muscle

MSO: maximum stimulator output

Mmax: maximum M-wave

RMS: root mean square

EFD: early facilitation delay 
SEM: standard error of the mean

\section{References}

Baker, S.N. (2011) The primate reticulospinal tract, hand function and functional recovery. $J$ Physiol, 589, 5603-5612.

Benjamini, Y. \& Hochberg, Y. (1995) Controlling the False Discovery Rate: A Practical and Powerful Approach to Multiple Testing. Journal of the Royal Statistical Society, 57, 289-300.

Biabani, M., Aminitehrani, M., Zoghi, M., Farrell, M., Egan, G. \& Jaberzadeh, S. (2018) The effects of transcranial direct current stimulation on short-interval intracortical inhibition and intracortical facilitation: a systematic review and meta-analysis. Rev Neurosci, 29, 99-114.

Burke, D. (2016) Clinical uses of $\mathrm{H}$ reflexes of upper and lower limb muscles. Clinical Neurophysiology Practice, 1.

Cash, R.F., Noda, Y., Zomorrodi, R., Radhu, N., Farzan, F., Rajji, T.K., Fitzgerald, P.B., Chen, R., Daskalakis, Z.J. \& Blumberger, D.M. (2017) Characterization of Glutamatergic and GABAA-Mediated Neurotransmission in Motor and Dorsolateral Prefrontal Cortex Using Paired-Pulse TMS-EEG. Neuropsychopharmacology, 42, 502-511. 
Chen, R., Tam, A., Butefisch, C., Corwell, B., Ziemann, U., Rothwell, J.C. \& Cohen, L.G. (1998) Intracortical inhibition and facilitation in different representations of the human motor cortex. $J$ Neurophysiol., 80, 2870-2881.

Cowan, J.M., Day, B.L., Marsden, C. \& Rothwell, J.C. (1986) The effect of percutaneous motor cortex stimulation on $\mathrm{H}$ reflexes in muscles of the arm and leg in intact man. J Physiol, 377, 333-347.

Crone, C. \& Nielsen, J. (1989) Methodological implications of the post activation depression of the soleus H-reflex in man. Exp.Brain Res., 78, 28-32.

Day, B.L., Dressler, D., Maertens, d.N., Marsden, C.D., Nakashima, K., Rothwell, J.C. \& Thompson, P.D. (1989) Electric and magnetic stimulation of human motor cortex: surface EMG and single motor unit responses. J Physiol, 412, 449-473.

Di Lazzaro, V., Pilato, F., Oliviero, A., Dileone, M., Saturno, E., Mazzone, P., Insola, A., Profice, P., Ranieri, F., Capone, F., Tonali, P.A. \& Rothwell, J.C. (2006) Origin of facilitation of motorevoked potentials after paired magnetic stimulation: direct recording of epidural activity in conscious humans. J Neurophysiol, 96, 1765-1771.

Di Lazzaro, V., Profice, P., Ranieri, F., Capone, F., Dileone, M., Oliviero, A. \& Pilato, F. (2012) I-wave origin and modulation. Brain Stimul, 5, 512-525. 
Di Lazzaro, V., Rothwell, J. \& Capogna, M. (2017) Noninvasive Stimulation of the Human Brain: Activation of Multiple Cortical Circuits. Neuroscientist, 1073858417717660.

Di Lazzaro, V., Ziemann, U. \& Lemon, R.N. (2008) State of the art: Physiology of transcranial motor cortex stimulation. Brain Stimul, 1, 345-362.

Dum, R.P. \& Strick, P.L. (2005) Frontal lobe inputs to the digit representations of the motor areas on the lateral surface of the hemisphere. J Neurosci, 25, 1375-1386.

Guzman-Lopez, J., Costa, J., Selvi, A., Barraza, G., Casanova-Molla, J. \& Valls-Sole, J. (2012) The effects of transcranial magnetic stimulation on vibratory-induced presynaptic inhibition of the soleus H reflex. Exp Brain Res, 220, 223-230.

Hanajima, R., Wang, R., Nakatani-Enomoto, S., Hamada, M., Terao, Y., Furubayashi, T., Okabe, S., Inomata-Terada, S., Yugeta, A., Rothwell, J.C. \& Ugawa, Y. (2007) Comparison of different methods for estimating motor threshold with transcranial magnetic stimulation. Clin Neurophysiol, 118, 2120-2122.

Iles, J.F. \& Pisini, J.V. (1992) Cortical modulation of transmission in spinal reflex pathways of man. J.Physiol, 455, 425-446. 
Kujirai, T., Caramia, M.D., Rothwell, J.C., Day, B.L., Thompson, P.D., Ferbert, A., Wroe, S., Asselman, P. \& Marsden, C.D. (1993) Corticocortical inhibition in human motor cortex. J Physiol, 471, 501-519.

Lemon, R.N., Maier, M.A., Armand, J., Kirkwood, P.A. \& Yang, H.W. (2002) Functional differences in corticospinal projections from macaque primary motor cortex and supplementary motor area. Adv Exp Med Biol, 508, 425-434.

Leukel, C., Taube, W., Rittweger, J., Gollhofer, A., Ducos, M., Weber, T. \& Lundbye-Jensen, J. (2015) Changes in corticospinal transmission following 8weeks of ankle joint immobilization. Clin Neurophysiol, 126, 131-139.

Marchand-Pauvert, V., Nicolas, G., Burke, D. \& Pierrot-Deseilligny, E. (2002) Suppression of the $\mathrm{H}$ reflex in humans by disynaptic autogenetic inhibitory pathways activated by the test volley. J.Physiol, 542, 963-976.

Meunier, S. (1999) Modulation by corticospinal volleys of presynaptic inhibition to la afferents in man. J.Physiol Paris, 93, 387-394.

Nielsen, J., Petersen, N. \& Ballegaard, M. (1995) Latency of effects evoked by electrical and magnetic brain stimulation in lower limb motoneurones in man. J Physiol, 484, 791-802. 
Nielsen, J., Petersen, N., Deuschl, G. \& Ballegaard, M. (1993) Task-related changes in the effect of magnetic brain stimulation on spinal neurones in man. J Physiol, 471, 223-243.

Niemann, N., Wiegel, P., Kurz, A., Rothwell, J.C. \& Leukel, C. (2017) Assessing TMS-induced D- and I-waves with spinal H-reflexes. J Neurophysiol. (published online ahead of print).

Pierrot-Deseilligny, E. \& Burke, D. (2005) The circuitry of the human spinal cord - Its role in motor control and movement disorders. Cambridge University Press, New York.

Rossini, P.M., Burke, D., Chen, R., Cohen, L.G., Daskalakis, Z., Di lorio, R., Di Lazzaro, V., Ferreri, F., Fitzgerald, P.B., George, M.S., Hallett, M., Lefaucheur, J.P., Langguth, B., Matsumoto, H., Miniussi, C., Nitsche, M.A., Pascual-Leone, A., Paulus, W., Rossi, S., Rothwell, J.C., Siebner, H.R., Ugawa, Y., Walsh, V. \& Ziemann, U. (2015) Non-invasive electrical and magnetic stimulation of the brain, spinal cord, roots and peripheral nerves: Basic principles and procedures for routine clinical and research application. An updated report from an I.F.C.N. Committee. Clin Neurophysiol, 126, 1071-1107.

Taube, W., Leukel, C., Nielsen, J.B. \& Lundbye-Jensen, J. (2015) Repetitive activation of the corticospinal pathway by means of rTMS may reduce the efficiency of corticomotoneuronal synapses. Cereb Cortex, 25, 1629-1637.

van der Linden, C. \& Bruggeman, R. (1993) Multiple descending corticospinal volleys demonstrated by changes of the wrist flexor $\mathrm{H}$-reflex to magnetic motor cortex stimulation in intact human subjects. Muscle Nerve, 16, 374-378. 
Ziemann, U., Reis, J., Schwenkreis, P., Rosanova, M., Strafella, A., Badawy, R. \& MullerDahlhaus, F. (2015) TMS and drugs revisited 2014. Clin Neurophysiol, 126, 1847-1868.

Ziemann, U., Rothwell, J.C. \& Ridding, M.C. (1996) Interaction between intracortical inhibition and facilitation in human motor cortex. J Physiol, 496, 873-881.

\section{Figure legends}

Figure 1. A: the left side depicts single trial EMG plots of conditioned $\mathrm{H}$-reflexes at EFD $0 \mathrm{~ms}$ (red) and plots of unconditioned H-reflexes (black) in a single subject. H-reflex onset in this subject was at $16.0 \mathrm{~ms}$ after electrically stimulating the median nerve. Note that the stimulus artefact from electrical stimulation was truncated. The right side shows the selected time frame (grey box) amplified.

B: displays the mean values of the single traces displayed in A (same subject). C: displays the mean traces of conditioned H-reflexes at EFD $-2 \mathrm{~ms},-1 \mathrm{~ms}$, and $0 \mathrm{~ms}$, and mean traces of unconditioned $\mathrm{H}$-reflexes (same subject as in A and B).

Figure 2. Upper part: shows peak-to-peak amplitude grand mean values and SEM of the Hreflex facilitation from S2 (orange), S1 (blue), S1-S2 (green). Post-hoc corrected results from paired Student's t-tests are displayed in the tables below the graphs. Green shadowed boxes indicate significant differences between S1-S2 and S2 according to the corrected significance level (correct.). Note that, for SOL, the mean values at EFDs $-2,-1$, and +10 ms do not include data from all participants. This was because in four participants the early facilitation occurred 
at a more negative ISIs than $-3 \mathrm{~ms}$ or a more positive ISI than $-2 \mathrm{~ms}$. Values from two participants are missing at EFD $-2 \mathrm{~ms}$, the value from one participant is missing at EFD $-1 \mathrm{~ms}$, and values from two participants are missing at EFD $+10 \mathrm{~ms}$.

Lower part: displays bivariate correlations of the initial period (RMS of $1 \mathrm{~ms}$ ) of the facilitation of the conditioned H-reflex (S1-S2 minus S2) between time points EFD -1 ms and EFD 0 ms. Black lines indicate linear regression of the data.

Figure 3. Left part: Displays grand mean values and SEM of S2, S1, and S1-S2 peak-to-peak MEPs. Right part: Displays individual differences in MEP values between S1-S2 and S2 (S1S2 MEP minus S2 MEP) for FCR and SOL. 